\title{
Factors Associated with Institutional Delivery in Boricha District of Sidama Zone, Southern Ethiopia
}

\author{
Tafese Tadele $^{1}$, Alemu Tamiso ${ }^{2}$, Tariku Tadele ${ }^{3}$ \\ ${ }^{1}$ Department of Public Health, Yirgalem Medical Science College, Southern Ethiopia \\ ${ }^{2}$ Unit of Epidemiology, Department of Public Health, College of Medicine and Health Science, \\ Arba Minch University, Ethiopia \\ ${ }^{3}$ Departement of Internal Medicine, College of Medicine and Health science, Hawassa University, Ethiopia
}

\begin{abstract}
Article Info
Article history:

Received Sept 20, 2014

Revised Oct 21, 2014

Accepted Nov 16, 2014

\section{Keyword:}

Factors associated Institutional delivery Prevalence Southern Ethiopia

ABSTRACT

Every year, 40 million women give birth at home without the help of a skilled birth attendant. In 2011, 287,000 women died during pregnancy or childbirth. Almost all these deaths occur in developing countries where mothers and children lack access to basic health care. Reports showed the low utilization of health facility for delivery service in Ethiopia. This study aimed to determine the utilization and factors influencing institutional delivery. Community based cross sectional study was conducted from January to February 2013/14 in Boricha District of Southern Ethiopia among mothers who gave birth in the last 1 year. Multistage sampling techniques were used to collect data from 546 mothers. Taking in to account place of birth for the last child, only $4.9 \%$ women gave birth in a health facility. Women's education level (AOR=4.4 (95\% CI=1.36-14.33)), timing of firstANC visit $(\mathrm{AOR}=.03(95 \% \mathrm{CI}=0.004-0.205))$, women's advice to deliver in a health facility during ANC (AOR = 31.15 (95\% CI=2.02479.52)), women's knowledge of birth related complications (AOR= 12.4 (95\% CI=2.67-57.16) ) and decision making power (AOR=0.2 (95\% CI=0.06$0.82)$ ) showed significant association with institutionional delivery. Institutional delivery in the study area was found to be very low. Raising awareness on institutional delivery to maximize delivery service utilization and strengthening provision of education and counseling to deliver in health facility during antenatal care visits at individual and community level should be given due emphasis.
\end{abstract}

\section{Corresponding Author:}

Alemu Tamiso,

Department Public Health,

College of Medicine and Health Sciences,

Arba Minch University, Southern Ethiopia.

Email: alemutamiso@yahoo.com

\section{INTRODUCTION}

A substantial reduction in maternal deaths has previously been noted from 543000 deaths in 1990 to an estimated 287000 by 2010, with a global rate of decline in the maternal mortality ratio of $3.1 \%$ per annum over the same period. Nevertheless, this rate of decline would now need to double in order to achieve the MDG target of reducing the maternal mortality ratio by three quarters between 1990 and 2015 [1].

Appropriate delivery care is crucial for both maternal and perinatal health and increasing skilled attendance at birth is a central goal of the safe motherhood and child survival movements. Skilled attendance at delivery is an important indicator in monitoring progress towards Millennium Development Goal 5 to reduce the maternal mortality ratio by three quarters between 1990 and 2015 [2]. WHO definition of skilled 
attendant is a health provider who has at least the minimum knowledge and skills to manage normal childbirth and provide basic (first line) emergency obstetric care [3].

Every year, 40 million women give birth at home without the help of a skilled birth attendant. In 2011, 287,000 women died during pregnancy or childbirth, and 6.9 million children died before reaching their fifth birthday. Almost all these deaths occur in developing countries where mothers and children lack access to basic health care [4].

According to EDHS 2011 an overwhelming majority of births (90 \%) in the five years before the survey were delivered at home; ten percent of births were delivered in a health facility [5]. In one survey conducted in the four regions of Ethiopia, 91\% of women with children 0-11 months of age reported that they delivered their last child at home. The survey also showed very low prevalence of professionally assisted delivery in all the four regions at $8 \%-24 \%$ in Tigray, 9\% in Oromiya and SNNPR, and as low as $4 \%$ in Amhara [6].

Another study done in Ethiopia shows, 16\% of deliveries were assisted by health professionals, while a significant majority (78\%) was attended by traditional birth attendants. The most important reasons for not seeking institutional delivery were the belief that it is not necessary (42\%) and not customary (36\%), followed by high cost (22\%) and distance or lack of transportation (8\%) [7].

A study done in North West of Ethiopia the common reasons for home delivery were closer attention from family members and relatives (60.9\%), home delivery is usual practice (57.7\%), unexpected labour (33.4\%), not being sick or no problem at the time of delivery (21.6\%) and family influence (14.4\%) [8].

The EDHS 2011 report showed the low utilization of health facility for delivery service which is only 6.2\% in SNNPR and 2011 annual report of Sidama zone health department was 4.3\% [5],[9]. Therefore, this study aimed to determine the utilization and identify the factors influencing use of delivery service in health facility by women in the study area with the view to propose appropriate intervention at the community and facility level to improve delivery service utilization.

\section{RESEARCH METHOD}

Study design: A community-based cross sectional study was conducted from January to February 2013/14 among all women of childbearing age (15 - 49 years) who gave at least one birth in the last one year preceding the study in Boricha District, Southern Nation and Nationality People Regional State, Ethiopia.

Sample size and sampling techniques: The sample size was calculated for prevalence of delivery in health facilities and the factors affecting institutional delivery. However, the sample size calculated by using the factors was smaller than the sample size obtained by using the prevalence of delivery in health facilities. Therefore the sample size obtained by using single population proportion was used,which was 546 .

The multistage and simple random sampling techniques were used. The District has one urban and eight rural sub-localities. The urban sub-locality has three kebeles and each rural sub-locality have four to five kebeles. Therefore one kebeles from urban and three sub-localities from the rural were selected randomly to include in the study.

First, a census was conducted in the selected kebeles. The eligible population was identified and a sampling frame which enlists all eligible study subjects was prepared. Then study participants were selected from the sampling frame using simple random sampling proportional to population size of each kebele.

Study population: All women of reproductive age who gave birth within the last one year in the household were given equal chance of being included in the study, and one woman of reproductive age sampled by the lottery method was included in the study.

Data collection: To maintain the quality of data, the questionnaire was adapted from previous study and pre-tested in the adjacent kebeles and necessary adjustments were made. Ten health extension workers from other kebeles who were fluent in the local language and fifteen community health promoters from their own kebele with the role of guide collected the data. Face to face interview technique was used in order to collect the data.

Each questionnaire was checked visually for completeness. The data was entered using EPI INFO version 3.5.1 and exported to SPSS version 16.0 for data cleaning and analysis. After data was entered cleaning was done by going back to the hard copies of the questionnaires.

\section{Data processing and analysis:}

The data was cleaned to check for its completeness, consistency and the presence of missed values and variables. Any error identified was corrected as necessary. Then, it was entered into a pre-designed format in Epi-info version 3.5.1 and transferred to SPSS version 16 for further analysis. Frequency, mean and 
standard deviation were done. Binary Logistic regression Model was fitted. First bivariate analysis between independent and dependent variables was done, then all independent variables that showed statistical significance with a p-value $<0.2$ in the bivariate analysis was included in the multivariate model. Those determinant with $\mathrm{p}$-value $<0.05$, in the multivariate analysis, were considered as independent and significant factors associated withinstitutional delivery.

Ethical consideration: This study protocol was reviewed and approved bythe research and publication office of HU School of public and Environmental Health and Addis continental Institute of public health. Permission to carry out the study was also granted from Sidama zone health department. Individual informed verbal consent was obtained after brief explanation of the purpose and benefits of the study to each respondent.

\section{RESULT AND ANALYSIS}

\subsection{Socio demographic characterstics}

A total of 546 women who gave birth within one year prior to the survey were interviewed. Their ages ranged from 15 to 44 years with mean and standard deviation age of $28.34 \pm 4.74$. Almost three quarters (72\%) of the respondents had never been to school and of those who reported to have attended formal schools; almost few (20.1\%) reported only seven years of schooling. Major occupation of respondents was housewife, accounting for $99.6 \%$ of the respondents. All of them (100\%) were married. The highest parity was nine (Table 1).

Table 1. Socio-demographic characteristics of the respondents who gave birth in 1 year period in Borichaworeda, Ethiopia, 2013/14 (N= 546)

\begin{tabular}{lcc}
\hline \multicolumn{1}{c}{ Variables } & Frequency & Percent (\%) \\
\hline Age inyears & 5 & 0.9 \\
$15-19$ & 124 & 22.7 \\
$20-24$ & 205 & 37.5 \\
$25-29$ & 144 & 26.4 \\
$30-34$ & 64 & 11.7 \\
$35-39$ & 4 & 0.7 \\
$40-44$ & $28.34 \pm 4.74$ & \\
Mean I SD & & 72.0 \\
Maternal education & 393 & 20.1 \\
Illiterate and only able to read and write & 110 & 7.9 \\
Grade 1-6 & 43 & 57.1 \\
Grade 7-10 & & 28.4 \\
Husband's highest educational level & 312 & 12.2 \\
Illiterate and able to read and write & 155 & 1.6 \\
Grade 1-6 & 66 & 0.7 \\
Grade 7-8 & 9 & \\
Grade 9-12 & & 66.5 \\
TEVT/Preparatory & & 30.5 \\
Respondents'monthlyincome & & 1.5 \\
<500 & & 1.5 \\
501-1000 & & \\
1001-1500 & & \\
\hline -1501 & 363 & \\
\hline
\end{tabular}

\subsection{Reproductive charactersics}

Two hundred thirteen (39\%) of the respondents have attended antenatal care (ANC) at least once in their gestation period. (Table 2) Very few, (4.9\%) of respondents reported to have delivered in a health facility in their most recent delivery. Of these, twenty four (4.4\%) delivered in health centers and three (0.5\%) delivered in a hospital. Among women who delivered at home, 32.4\% were assisted by untrained neighbors, $37.8 \%$ were assisted by relatives, $16.8 \%$ were assisted by Traditional Birth Attendants (TBA's) while $12.1 \%$ were assisted by husband's mother and the rest $0.8 \%$ were Self assisted births (Table.2). 
Table 2. Reproductive health practices among women who gave birth in 1 year period in Borichaworeda, Ethiopia, 2013/14. ( $\mathrm{N}=546)$

\begin{tabular}{lcc}
\hline \multicolumn{1}{c}{ Variables } & Frequency & Percent (\%) \\
\hline Women who have received ANC & 213 & 39.0 \\
Setting in which delivery was conducted & & \\
Health center & 24 & 4.4 \\
Hospital & 3 & 0.5 \\
At home & 519 & 95.1 \\
Birth assistance at home & & \\
TBA & 87 & 16.8 \\
Neighbors & 168 & 32.4 \\
Husband's mother & 63 & 12.1 \\
Relatives & 197 & 37.9 \\
Self assisted & 4 & 0.8
\end{tabular}

\subsection{Prevalence of institutional delivery}

Majority (97.3\%) of the respondents reported that they know the availability of delivery service in health institutions. The reasons given for not getting delivery service from health institution were more than a quarter 505 (97.3\%) respondents reported labor is short and ease for them, 14 (2.7\%) women mentioned that they didn't know the availability of institutional delivery service in health facility and very few $9(1.7 \%)$ women revealed that health institution is too far to utilize delivery service.

From those who delivered at home three hundred seventy four (68.5\%) women gave birth at a place where they planned ahead. Of the total respondents more than half 335(61.4\%) women revealed that they prefer to give birth at home and only $211(38.6 \%)$ women prefer to deliver in health facility with skilled birth attendant. Almost all $61.4 \%$ of the respondents revealed that they will have care from families during child birth.

\subsection{Factors associated with institutional delivery}

The various factors associated with utilization of institutional delivery services are shown on table 3. In the bivariate analysis; women's age, women's education, women's prior history of abortion, the index pregnancy being planned, timing of ANC first visit, women's advice to deliver in health institution during ANC, women's knowledge of birth related complications and decision making power were significantly associated with utilization of delivery service. Younger women were 0.106 times more likely to utilize health institution during labour and delivery unlike the older women.

The relationship between place of delivery and women's education shows that the use of health facilities increases with an increase in the educational level of women. It is revealed that women with primary school educational level were almost twice as likely to use formal health facilities for their place of delivery as women with illiterate and only able to read and write. On the other hand, table 4 showed that years spent in school showed a significant association with seeking of skilled care during delivery from health institution with women who have educational attainment of grade 7-10 having a higher proportion of deliveries (27.9\%) attended by skilled personnel in health facility compared to those with fewer schooling years or those who did not go to formal schooling.

Women who give high value to the expected child may be more likely to utilize health facility at delivery than those with unwanted pregnancies (OR. 9.64(95\% CI=2.26- 41.13)).

Women who started ANC clinic late wereless likely utilized health institution during delivery to be assisted by a skilled attendant compared to those who started early (OR=0.037 (95\% CI=0.013- 0.105)). (Table 3)

Women who were advised during ANC by health workers to deliver in a health facility had a higher proportion delivering in health institution with a skilled attendant compared to those who were not.( Table 3) Proportion of women who delivered in health institution with skilled care at delivery increased with knowledge of birth related complications from 1.4\% among women who did not mention any to $8.7 \%$ among those who mentioned birth related complications $(p<.001)$. Women who reported that they had previous history of abortion also have an increased chance to use health facilities for delivery compared to those who had no previous history of abortion (OR 8.85 (95\%CI (2.58-30.36)). The finding of this study showed husband's decision making power is less likely associated with utilization of health facility for delivery compared to decision making power of both (95\% CI=0.075- 0.544). (Table 3)

All variables which showed a significant association with place of delivery care in the bivariate analysis were put in a multiple logistic regression model by splitting the variables in to two groups based on the presence or absence of ANC visit to assess individual variable effects on health institution delivery care during birth (Table 3).However, in the multivariate analysis five factors kept their significant associations with the use of delivery service from health facility. 
Even after controlling for confounding factors,women's education level(AOR=4.41 (95\% CI=1.3614.33)), timing of ANC first visit (AOR= .029 (95\% CI=.004- .205)), women's advice to deliver in a health facility during ANC (AOR = 31.15 (95\% CI=2.02- 479.52)),women's knowledge of birth related complications $(\mathrm{AOR}=12.36(95 \% \mathrm{CI}=2.67-57.16))$ and decision making power $(\mathrm{AOR}=.219(95 \% \mathrm{CI}=.058-$ .824)) showed significant association with use of health institution at delivery. (Table 3)

The dependent variable which has been used in the bivariate analysis is also included in the multiple logistic regression analysis. This variable is classified as dichotomous response variable which is assigned the values 0 and 1 . Regarding the independent variables, only those variables which show significant relationships with institutional delivery service utilization are included in the regression analysis. In order to determine the association between each independent variable and place of delivery two multiple logistic models were used. The main consideration here is the large number of missing responses. Those respondents who do not have ANC visit about 333 were not asked for the services provided during ANC visit and they are classified as system missing. Therefore analysis was made by using two multivariate models one by including variables of ANC $(\mathrm{N}=213)$ and another by excluding them $(\mathrm{N}=546)$. Therefore women's age, maternal education, history of abortion, the last pregnancy being planned, timing of ANC first visit, advice to deliver in health institution during ANC, knowledge of birth related complications and decision maker on place of delivery entered in to one model and all variables except timing of ANC first visit and advice to deliver in health institution during ANC in to another model (Table 3).

Table 3. Socio demographic and Reproductive health determinants associated with delivery services utilization by women who gave birth in 1 year period in Borichaworeda, Ethiopia, 2013/14

\begin{tabular}{|c|c|c|c|c|}
\hline \multirow{3}{*}{ Variable } & \multicolumn{2}{|c|}{ Place of delivery } & \multirow{3}{*}{ COR(95\% CI) } & \multirow{3}{*}{ AOR (95\% CI) } \\
\hline & Home delivery & institution birth & & \\
\hline & № (\%) & № (\%) & & \\
\hline \multicolumn{5}{|l|}{ Women's age } \\
\hline 15-19 & $2(40.0 \%)$ & $3(60.0 \%)$ & 1.00 & 1.00 \\
\hline $20-24$ & 107(86.3\%) & $17(13.7 \%)$ & $0.106(.016-.681) *$ & $0.005(.000-.119)$ \\
\hline $25-29$ & 199(97.1\%) & $6(2.9 \%)$ & $0.020(.003-.143) *$ & $0.002(.000-.040)$ \\
\hline $30-34$ & $143(99.3 \%)$ & $1(0.7 \%)$ & $0.005(.000-.067) *$ & $0.000(.000-.017)$ \\
\hline $35-39$ & $64(100.0 \%)$ & 0 & & \\
\hline $40-44$ & $4(100.0 \%)$ & 0 & & \\
\hline \multicolumn{5}{|l|}{ Maternal Education } \\
\hline $\begin{array}{l}\text { Illiterate and only able to read } \\
\text { and write }\end{array}$ & $384(97.7 \%)$ & $9(2.3 \%)$ & 1.00 & 1.00 \\
\hline Grade 1-6 & $104(94.5 \%)$ & $6(5.5 \%)$ & $2.46(0.857-7.073)$ & $1.02(0.26-4.00)$ \\
\hline Grade 7-10 & $31(72.1 \%)$ & $12(27.9 \%)$ & $16.52(6.46-42.22) * *$ & $4.41(1.36-14.33) *$ \\
\hline \multicolumn{5}{|l|}{ Timing of ANC first visit } \\
\hline $1-3$ month/s & $8(33.3 \%)$ & $16(66.7 \%)$ & 1.00 & 1.00 \\
\hline 4-6 months & $149(93.1 \%)$ & $11(6.9 \%)$ & $\begin{array}{c}0.037(0.013-0.105) * \\
*\end{array}$ & $.029(0.004-0.205) * *$ \\
\hline 7-9 months & $29(100.0 \%)$ & 0 & & \\
\hline \multicolumn{5}{|l|}{$\begin{array}{l}\text { Advice to deliver in health } \\
\text { institution during ANC }\end{array}$} \\
\hline NO & $171(90.5 \%)$ & $18(9.5 \%)$ & 1.00 & 1.00 \\
\hline Yes & $7(50.0 \%)$ & $7(50.0 \%)$ & $9.50(2.99-30.15) * *$ & $31.15(2.02-479.52) *$ \\
\hline I don't remember & $8(80.0 \%)$ & $2(20.0 \%)$ & $2.38(0.47-12.05)$ & $1.17(0.056-24.62)$ \\
\hline \multicolumn{5}{|l|}{ History of abortion } \\
\hline No & $509(95.7 \%)$ & $23(4.3 \%)$ & 1.00 & \\
\hline Yes & $10(71.4 \%)$ & $4(28.6 \%)$ & $8.85(2.58-30.36) * *$ & 3.70(0.599- 22.89) \\
\hline \multicolumn{5}{|l|}{$\begin{array}{l}\text { The last pregnancy being } \\
\text { planned }\end{array}$} \\
\hline No & 226(99.1\%) & $2(.9 \%)$ & 1.00 & 1.00 \\
\hline Yes & $293(92.1 \%)$ & $25(7.9 \%)$ & $9.64(2.26-41.13) *$ & 4.29 (0.89-20.63) \\
\hline \multicolumn{5}{|l|}{$\begin{array}{l}\text { Knowledge of birth related } \\
\text { complications }\end{array}$} \\
\hline No & $278(98.6 \%)$ & $4(1.4 \%)$ & 1.00 & 1.00 \\
\hline Yes & $241(91.3 \%)$ & $23(8.7 \%)$ & $6.63(2.26-19.45) * *$ & $12.36(2.67-57.16) *$ \\
\hline \multicolumn{5}{|l|}{ Decision maker } \\
\hline Both of us & $222(91.0 \%)$ & $22(9.0 \%)$ & 1.00 & 1.00 \\
\hline My husband & $249(98.0 \%)$ & $5(2.0 \%)$ & $0.203(0.075-0.544) *$ & $0.219(0.058-0.824) *$ \\
\hline Myself & $42(100.0 \%)$ & 0 & & \\
\hline Husband's family & $6(100.0 \%)$ & 0 & & \\
\hline
\end{tabular}

$\mathrm{CI}=$ Confidence Interval, OR $=$ Odds ratio; $*$ Significant at $\mathrm{p}<0.05, * *$ significant at $\mathrm{p}<0.001$, Adjusted for all the independent variables indicated in the table 


\section{DISCUSSION}

Findings of this study showed that only few of the respondents had delivered in health institution by a skilled provider. This is lower compared to the report of Ethiopian Demographic and Health Survey (EDHS) 2011 result for SNNPR (6.2\%) (5) and FMOH report of 2010 which indicate less than 16.8\% [10]. This could be because the DHS and FMOH report includes the urban areas where considerably a large number of deliveries attended by health professionals. This estimate compares well with Amhara region (4.7\%) observed in the last 10 kilometer base line house hold survey 2009 but lower compared to the rates obtained from the study region (9\%) in the same survey (6). Our finding is also less than the findings from study conducted in different part of Ethiopia $16 \%, 19.6 \%, 12.3 \%, 12.1 \%$ and $16 \%$ on programmatic correlate of maternal health care seeking behaviors in Ethiopia, utilization of antenatal care services among teenagers in Ethiopia, Munisa woreda South East of Ethiopia, North West of Ethiopia and Kembatta-Tembaro zone of South Ethiopia respectively [7],[8],[11]-[13]. And it is also far less than findings fromUganda (45.4\%), Nigeria (43.5\%), Kenya (42.6\%), India (44\%) and Pakistan (41\%) [14]-[18]. This could be due to the difference in maternal characteristics and the setup.

The Majority (97.3\%) of women who delivered their infants at home commonly mentioned labour is short and ease for them. This finding was higher than cross sectional study done in North West Ethiopia $33.4 \%$ said labor was short/ urgent and $21.6 \%$ said they did not have any problem to go to health facilities [8].

This study revealed $39 \%$ of the respondents had attended ANC at least once in their gestational period. This finding was in line with the study done in MunisaDistrict of South East Ethiopia which revealed $34.7 \%$ of mothers had at least one ANC visit during their last pregnancy one year prior to the study period. But it is not consistent with finding from Kembatta-Tembaro of SNNPR and Sekela district of North West of Ethiopia which was $71 \%$ and $66.8 \%$ respectively $(7,8)$. And it was also not similar with the study done in Lubumbashi city of Democratic Republic of Congo - 92.6\% and Kenya demographic and health survey result which is 92.6\% [19],[16]. The inconsistency of the findings could be due to maternal characters and resident different.

In this study a number of important socio demographic and reproductive health factors were found to have a significant influence on utilization of institutional delivery services at child birth. It include women's education, women's advice to deliver in health institution during ANC, timing of ANC first visit, women's knowledge of birth related complications and decision making power on place of delivery. The strongest association was found in timing of ANC first visit, which was also true in the bivariate analysis.

In this study literate mothers were more likely use health facility during delivery than illiterate and only able to read and write. Studies done in MunisaDistrict of South East Ethiopia, Sekela district of North West Ethiopia and India shows similar finding which is maternal educational level have strong positive association with place of delivery [8],[9],[20]. In study done in Munisaworeda, husband's education shows a strong and significant association with the utilization of institutional delivery services [13] but this finding didn't showed the association with health institution delivery. It could be due to the vast majority of respondents were being illiterate.

The finding of our study showed twelve (27.9\%) women with educational attainment of grade 7-10 utilized health facility during delivery of their index child. The results of this study are similar compared to the report of Kenya Demographic and Health Survey (KDHS) 2008/09 result (27\%) (16).This is far less than findings from Nigeria's (75.8\%) [17]. this could be differences in the local contexts.

Women who were knowledgeable of birth related complications were more likely to utilize health facilities for delivery compared to those with no knowledge (AOR=12.36 (95\% CI=2.67-57.16)). The finding of our study showed $8.7 \%$ women who have knowledge of birth related complications gave birth in health institution. The finding of this study is consistent with Tanzania's (7.4 \%) [21] and less than Kenya's (18.5\%) [22]. It is expected that a better informed individual is better placed to make reasonable decisions.

The strength of this study includes: it is a community based; census was conducted before data collection to identify women who gave birth in the last one year and also large sample size was used. Limitations of the study are: there could be social desirability bias; to reduce this we used health extension workers from other kebeles.

\section{CONCLUSION}

The study identified very low utilization of delivery services from health institutions in the area. Women's education, timing of ANC first visit, advice during ANC visit to deliver in health facility, knowledge of birth related complications and communal decision making role affected institutional delivery service utilization in the area.Raising awareness on institutional delivery to maximize delivery service 
utilization and strengthening provision of education and counseling to deliver in health facility during antenatal care visits at individual and community level should be given due emphasis.

\section{ACKNOWLEDGEMENTS:}

The authors are very grateful to Boricha district for its administrative and technical assistance. The school of public health and environmental science, college of medicine and health science, Hawassa University deserves acknowledgement for giving a chance and sidama zone health departments also thesaurus for financial support.

\section{REFERENCES}

[1] WHO, "World Health Statistics 2013”, World Health Organization, 2013.

[2] United Nations, “The Millennium Development Goals Report 2005”, New York: United Nations, 2006. [http://www.un.org/milleniumgoals/].

[3] WHO, "Skilled birth attendant", World Health Organization, Nepal, 2006. http://www.nep.searo.who.int/LinkFiles/Home_Skilled_Birth_Attendant1.pdf

[4] Save the Children, "Surveying the First Day, State of the World's Mother 2013", 2013

[5] Central Statistical Agency [Ethiopia] and ORC Macro, "Ethiopia Demographic and Health Survey 2011", Addis Ababa, Ethiopia and Calverton, Maryland, USA: Central Statistical Agency and ORC Macro, 2011.

[6] The last ten kilometers project. Baseline house hold health survey, "Amhara, Oromia, SNNPR and Tigray”, JSI Research and training, Inc., Addis Ababa, Ethiopia, 2009.

[7] Shiferaw et al., "Why do women prefer home births in Ethiopia?”, BMC Pregnancy and Childbirth, vol. 13, pp. 5, 2013.

[8] Teferra et al., "Institutional delivery service utilization and associated factors among mothers who gave birth in the last 12 months in Sekela District, North West of Ethiopia: A community - based cross sectional study”, BMC Pregnancy and Childbirth, vol. 12, pp. 74, 2012.

[9] Sidama Zone Health Department annual report, 2003E.C.

[10] Ethiopia Federal Ministry of Health, "Report on the safe motherhood”, Addis Ababa, Family Health Department, 2010.

[11] Ali Mehryar K, Wuleta B, Samuel Y, et.al., "Programmatic correlates of maternal healthcare seeking behaviors in Ethiopia”, Ethiop. J. Health Dev, vol/issue: 24(1), pp. 92-99, 2010.

[12] Tewodros A, Jemal H., Dereje H., "Utilization of antenatal care services among teenagers in Ethiopia”, Ethiop. J. Health Dev, vol/issue: 24(3), pp. 221-225, 2010.

[13] Amano et al., "Institutional delivery service utilization in Munisa Woreda, South East Ethiopia: a community based cross sectional study”, BMC Pregnancy and Childbirth, vol. 12, pp. 105, 2012.

[14] Anyait et al., "Predictors for health facility delivery in Busia district of Uganda: a cross sectional study", BMC Pregnancy and Childbirth, vol. 12, pp. 132, 2012.

[15] Sabine G., Oona C., "Still too far to walk: Literature review of the determinants of delivery service use", BMC Pregnancy and Childbirth, vol. 9, pp. 34, 2009.

[16] Kitui et al., "Factors influencing place of delivery for women in Kenya: an analysis of the Kenya demographic and health survey, 2008/2009”, BMC Pregnancy and Childbirth, vol. 13, pp. 40, 2013.

[17] Amardeep T, Amar M, Kaberi B, et.al., "Analysis of choice of delivery location from national survey in India", available at www.biomedcentral.com/1471-2458/8/29.

[18] Agha, Carton, “Determinants of institutional delivery in rural Jhang, Pakistan”, International Journal for Equity in Health, vol. 10, pp. 31, 2011.

[19] Ntambue et al., "Determinants of maternal health services utilization in urban settings of the Democratic Republic of Congo - A Case study of Lubumbashi City”, BMC Pregnancy and Childbirth, vol. 12, pp. 66, 2012.

[20] Deepthi S., "Increasing institutional delivery and access to emergency obstetric care services in rural Uttar Pradesh", Journal of Family walfare, vol.56, 2010.

[21] Rose M, Japhet K, Melkzedeck, Leshabari et.al., "Use pattern of maternal health services and determinants of skilled care during delivery in Southern Tanzania: implications for achievement of MDG-5 targets”, BMC Pregnancy and Childbirth, vol. 7, pp. 29, 2007.

[22] Carol W, Moses M, Evans M, et al., "Delivery practices and associated factors among mothers seeking child welfare services in selected health facilities in Nyandarua south district, Kenya. Wanjira et al”, BMC Public Health, vol. 11, pp. 360, 2011. 